\section{Effect of highly active antiretroviral therapy (HAART) on the natural history of ocular manifestations in HIV-infected children}

S Esposito', A Porta', J Bojanin', L Gualtieri',

L Cesati', E Vismara ${ }^{2}$ and N Principi'
${ }^{1}$ Institute of Pediatrics, University of Milan, Fondazione IRCCS 'Ospedale Maggiore Policlinico, Mangiagalli e Regina Elena', Milan, Italy

immune rect
symptom.

Eye (2006) 20, 595-597. doi:10.1038/sj.eye.6702189; published online 13 January 2006

Keywords: ocular manifestations; HIV; HAART; CMV; children

\section{Introduction}

Until recently, ophthalmic abnormalities were common in HIV-infected patients and often due to opportunistic infections. ${ }^{1-4}$ However, the introduction of highly active antiretroviral therapy (HAART) has led to a considerable decline in opportunistic infections and a reduction in HIV-related deaths. ${ }^{5,6}$ All of the available reports concerning ocular involvement in HIV-infected patients in the HAART era relate to adults; $;^{7-10}$ no childhood data are available. The aim of this study was to determine the effect of HAART on the natural history of ocular manifestations in HIV-infected children.

\section{Materials and methods}

We studied all of the HIV-infected children attending the Institute of Pediatrics, University of Milan, Milan, Italy, between 1982 and 2004.

Every 3 months, they were physically examined and underwent indirect fundoscopy conducted by an experienced ophthalmologist; if diagnosed as having eye disease, they were evaluated by the ophthalmologist every week
${ }^{2}$ Department of

Ophthalmology,

Fondazione IRCCS

'Ospedale Maggiore

Policlinico,

Mangiagalli e Regina Elena', Milan, Italy

Correspondence: N Principi, Institute of Pediatrics, University of Milan, Fondazione IRCCS 'Ospedale Magglore Policlinico, Mangiagalli e Regina Elena', Via Commenda 9, Milano 20122, Italy

Tel: + 390257992 498;

Fax: + 390250320226

E-mail: Nicola.Principi@

unimi.it

Received: 5 September 2005

Accepted in revised form: 7 October 2005

Published online: 13 January 2006

This study was conducted in accordance with the guidelines for human experimentation specified by the authors' institution

Presented in part at the $23 \mathrm{rd}$ Annual Meeting of the European Society for Paediatric Infectious Diseases-ESPID, Valencia, Spain, 18-20 May 2005 
while on specific therapy and monthly thereafter. On the basis of previously published criteria, ${ }^{8}$ ocular involvement was graded as: $0=$ no disease, $1=$ stable, $2=$ smoldering, and $3=$ active.

The study was approved by the Ethics Committee of the University of Milan, Milan, Italy, and written informed consent was obtained from the parents or legal guardians of the children.

The patients' case notes, the hospital's HIV database, and laboratory records were monthly reviewed and recorded. The clinical and laboratory findings before and after the introduction of HAART were compared. HAART was defined as any combination antiretroviral therapy containing a protease inhibitor. The date of the introduction of HAART was defined as 1 January 1998, this being the date that protease inhibitors were first used at our centre.

All of the data were analysed using SAS Windows v.12. A $P$-value of $<0.05$ was considered statistically significant.

\section{Results}

The cohort consisted of 117 HIV-infected children (61 males), with a follow-up ranging from 0.09 to 22.31 years (median, 16.33 years). A total of nine cases of ocular involvement (7.7\%) were diagnosed between 1983 and 1994, before the introduction of HAART (Table 1). The ocular manifestations were diagnosed in all cases during routine ophthalmologic screening in the absence of visual symptoms. Five of the patients with CMV retinitis showed granular retinitis with intraretinal haemorrhages, and three perivascular retinitis; in all eight children, CMV viremia was detected by polymerase chain reaction. The diagnosis of toxoplasmic retinochoroiditis was based on the presence of severe anterior chamber and vitreous inflammation, together with delicate white-greyish spots and haemorrhages on the ocular fundus, and the positive determination of anti-Toxoplasma gondii antibodies in blood.

Despite ganciclovir therapy and, in the case of toxoplasmosis, pyrimethamine and sulfadiazine, the ocular manifestations resolved only in the child without CD4 cell depletion; however, all nine children died 4-24 months (median, 15 months) after the diagnosis of ophthalmic disease.

No case of ocular involvement was observed after the introduction of HAART ( $P=0.011$ vs before HAART).

\section{Discussion}

This study demonstrates for the first time that the introduction of HAART has had a significant impact on 
the natural history of ocular manifestations in HIVinfected children, thus suggesting that a reduction in the frequency of ophthalmologic follow-up should be considered for HAART-treated HIV-infected children with immune reconstitution and no visual symptom.

The protective effect of HAART on ocular manifestations may be explained by the post-HAART reconstitution of T-lymphocyte responses against a variety of pathogens, including CMV and T. gondii. ${ }^{11}$ At the same time, paradoxical inflammatory complications attributed to post-HAART immune reconstitution have been frequently described in adults. ${ }^{11,12}$ However, no cases have been reported in childhood probably because of the small number of patients with ocular opportunistic infections since the introduction of HAART in paediatric age.

Low CD4 cell counts and high HIV RNA levels have been associated with ocular involvement and disease progression in HIV-infected adults. We have no HIV RNA data relating to our patients with ocular manifestations because all of them were followed up when viral load was not routinely measured. However, as the majority were severely immunosuppressed, and the only child with a favourable ocular outcome showed no CD4 cell depletion, we can confirm the importance of CD4 cell counts in ocular involvement, as well as in the favourable remission of disease.

Finally, it is still unknown how long the beneficial effect of HAART on the development of ocular involvement and the risk of progression after previous disease may be sustained. It is probable that CMV disease and other opportunistic infections will continue to evolve, and so further monitoring is required to document these changes.

\section{References}

1 Robinson MR, Ross ML, Whitcup SM. Ocular manifestations of HIV infection. Curr Opin Ophthalmol 1999; 10: 431-437.

2 Moraes Jr HV. Ocular manifestations of HIV/AIDS. Curr Opin Ophthalmol 2002; 13: 397-403.

3 Wren SME, Fielder AR, Bethell D, Lyall EGH, TudorWilliams G, Cocker KD et al. Cytomegalovirus retinitis in infancy. Eye 2004; 18: 389-392.

4 Kahraman G, Krepler K, Franz C, Ries E, Maar N, Wedrich A et al. Seven years of HAART impact on ophthalmic management of HIV-infected patients. Ocul Immunol Inflamm 2005; 13: 213-218.

5 Mocroft A, Ledergerber B, Katlama C, Kirk O, Reiss P, d'Arminio Monforte A et al. Decline in the AIDS and death rates in the EuroSIDA study: an observational study. Lancet 2003; 362: 22-29.

6 Jabs DA, Holbrook JT, Van Natta ML, Clark R, Jacobson MA, Kempen JH et al. Risk factors for mortality in patients with AIDS in the era of highly active antiretroviral therapy. Ophthalmology 2005; 112: 771-779.

7 Deayton J, Wilson P, Sabin CA, Davey CC, Johnson MA, Emery VC et al. Changes in the natural history of cytomegalovirus retinitis following the introduction of highly active antiretroviral therapy. AIDS 2000; 14: 1163-1170.

8 Lin DY, Warren JF, Lazzeroni LC, Wolitz RA, Mansour SE. Cytomegalovirus retinitis after initiation of highly active antiretroviral therapy in HIV-infected patients. Retina 2002; 22: $268-277$.

9 Ng WT, Versace P. Ocular association of HIV infection in the era of highly active antiretroviral therapy and the global perspective. Clin Exp Ophthalmol 2005; 33: 317-329.

10 Goldberg DE, Smithen LM, Angelilli A, Freeman WR. HIV-associated retinopathy in the HAART era. Retina 2005; 25: 633-649.

11 French MA, Price P, Stone SF. Immune restoration disease after antiretroviral therapy. AIDS 2004; 18: 1615-1628.

12 Miserocchi E, Modorati G, Brancato R. Immune recovery uveitis in an iatrogenically immunosuppressed patient. Eur J Ophthalmol 2005; 15: 510-512. 